\title{
Microvesicular Steatosis
}

National Cancer Institute

\section{Source}

National Cancer Institute. Microvesicular Steatosis. NCI Thesaurus. Code C82982.

A morphologic finding indicating intracytoplasmic fat accumulation in the liver parenchyma. Unlike macrovesicular steatosis, the fat accumulation occurs around the nucleus of the hepatocytes, without displacing it. 